\title{
Conformal Field Theory with Two Kinds of Bosonic Fields and Two Linear Dilatons
}

\author{
Davoud Kamani* \\ Faculty of Physics, Amirkabir University of Technology (Tehran Polytechnic) \\ P.O.Box: 15875-4413, Tehran, Iran
}

(Received on 26 June, 2009)

\begin{abstract}
We consider a two-dimensional conformal field theory which contains two kinds of the bosonic degrees of freedom. Two linear dilaton fields enable us to study a more general case. Various properties of the model such as OPEs, central charge, conformal properties of the fields and associated algebras will be studied.
\end{abstract}

Keywords: String; Linear Dilaton CFT; OPE.

\section{INTRODUCTION}

Among the various conformal field theories (CFTs) the linear dilaton CFT has some interesting applications in the string theory, e.g. see [1-6]. In this CFT, modification of the energy-momentum tensor was anticipated by a linear dilaton field. This CFT significantly changes the behavior of the worldsheet theory. In addition, this CFT is a consistent way for reducing the spacetime dimension without compactification. The linear dilaton CFT also appears as an ingredient in many string backgrounds, critical and non-critical. According to its importance we proceed to develop it.

In this paper we consider an action which is generalization of the bosonic part of the $\mathcal{N}=2$ superstring theory. In addition, we introduce two linear dilaton fields to build our model. Thus, we study a conformally invariant field theory in two flat dimensions. Anticipating to the string theory, we refer to these two dimensions as the string worldsheet. Various OPEs of the model will be calculated. Due to the dilaton fields, some of these OPEs, and also conformal transformations of the worldsheet fields $X^{\mu}(\sigma, \tau)$ and $Y^{\mu}(\sigma, \tau)$ have deviations from the standard forms. Presence of some parameters in the central charge enables us to receive a desirable dimension for the spacetime. The algebra of the oscillators reveals that the oscillators of $X$-fields do not commute with that of $Y$-fields.

This paper is organized as follows. In section 2, we shall introduce the action of the model and the linear dilatonic energy-momentum tensor associated to it. In section 3, various OPEs of the model will be studied. In section 4 , conformal transformations of the worldsheet fields will be obtained. In section 5, various quantities will be expressed in terms of the oscillators, and two algebras for the model will be obtained. Section 6 is devoted for the conclusions.

\section{THE MODEL}

We begin with the action of the scalar fields $X^{\mu}(z, \bar{z})$ and $Y^{\mu}(z, \bar{z})$ in two dimensions

$$
\begin{aligned}
S & =\frac{1}{2 \pi \alpha^{\prime}} \int d^{2} z\left(\partial_{z} X^{\mu} \partial_{\bar{z}} X_{\mu}+\beta \partial_{z} Y^{\mu} \partial_{\bar{z}} Y_{\mu}\right. \\
& \left.+\lambda\left(\partial_{z} X^{\mu} \partial_{\bar{z}} Y_{\mu}+\partial_{\bar{z}} X^{\mu} \partial_{z} Y_{\mu}\right)\right)
\end{aligned}
$$

where $\mu \in\{0,1, \ldots, D-1\}$ and $\beta$ and $\lambda$ are constants, i.e. independent of $z$ and $\bar{z}$. For the spacetime we consider the flat Minkowski metric $\eta_{\mu v}=\operatorname{diag}(-1,1, \ldots, 1)$. The special case $\beta=1$ and $\lambda=0$ indicates the bosonic part of the $\mathcal{X}=2$ super-conformal field theory. Thus, we say the set $\left\{X^{\mu}\right\}$ describes the spacetime coordinates. In other words, $X^{\mu}(\sigma, \tau)$ is regarded as the embedding of the worldsheet in the spacetime. However, $Y^{\mu}(\sigma, \tau)$ enters essentially in the same way. So the set $\left\{Y^{\mu}(\sigma, \tau)\right\}$ does not describe additional dimensions. The conformal invariance of this action, i.e. symmetry under the conformal transformations $z \rightarrow z^{\prime}(z)$ and $\bar{z} \rightarrow \bar{z}^{\prime}(\bar{z})$, leads to the zero conformal weights for the fields $X^{\mu}$ and $Y^{\mu}$.

The equations of motion, extracted from the action (1), are

$$
\begin{aligned}
& \partial_{z} \partial_{\bar{z}} X^{\mu}+\lambda \partial_{z} \partial_{\bar{z}} Y^{\mu}=0, \\
& \lambda \partial_{z} \partial_{\bar{z}} X^{\mu}+\beta \partial_{z} \partial_{\bar{z}} Y^{\mu}=0 .
\end{aligned}
$$

We assume that the determinant of the coefficients of these equations to be nonzero i.e.,

$$
\operatorname{det}\left(\begin{array}{cc}
1 & \lambda \\
\lambda & \beta
\end{array}\right)=\beta-\lambda^{2} \neq 0
$$

Therefore, we obtain

$$
\begin{aligned}
& \partial_{z} \partial_{\bar{z}} X^{\mu}=0 \\
& \partial_{z} \partial_{\bar{z}} Y^{\mu}=0 .
\end{aligned}
$$

These imply $\partial_{z} X^{\mu}$ and $\partial_{z} Y^{\mu}$ are functions of $z$, and $\partial_{\bar{z}} X^{\mu}$ and $\partial_{\bar{z}} Y^{\mu}$ are functions of $\bar{z}$.

The corresponding energy-momentum tensor has the components 


$$
\begin{aligned}
& T_{z z}^{\prime} \equiv T^{\prime}(z)=-\frac{1}{\alpha^{\prime}}\left(: \partial_{z} X^{\mu} \partial_{z} X_{\mu}:+\beta: \partial_{z} Y^{\mu} \partial_{z} Y_{\mu}:+\lambda: \partial_{z} X^{\mu} \partial_{z} Y_{\mu}:+\lambda: \partial_{z} Y^{\mu} \partial_{z} X_{\mu}:\right), \\
& T_{\bar{z} \bar{z}}^{\prime} \equiv \tilde{T}^{\prime}(\bar{z})=-\frac{1}{\alpha^{\prime}}\left(: \partial_{\bar{z}} X^{\mu} \partial_{\bar{z}} X_{\mu}:+\beta: \partial_{\bar{z}} Y^{\mu} \partial_{\bar{z}} Y_{\mu}:+\lambda: \partial_{\bar{z}} X^{\mu} \partial_{\bar{z}} Y_{\mu}:+\lambda: \partial_{\bar{z}} Y^{\mu} \partial_{\bar{z}} X_{\mu}:\right), \\
& T_{z \bar{z}}^{\prime}=T_{\bar{z} z}^{\prime}=0,
\end{aligned}
$$

where : : denotes normal ordering.

It is possible to construct a more general CFT with the same action (1), but with different energy-momentum tensor

$$
\begin{aligned}
& T(z)=\Lambda_{i j}: \partial_{z} X_{i}^{\mu} \partial_{z} X_{j \mu}:+V_{\mu}^{i} \partial_{z}^{2} X_{i}^{\mu}, \\
& \tilde{T}(\bar{z})=\Lambda_{i j}: \partial_{\bar{z}} X_{i}^{\mu} \partial_{\bar{z}} X_{j \mu}:+V_{\mu}^{i} \partial_{\bar{z}}^{2} X_{i}^{\mu}, \\
& T_{z \bar{z}}=T_{\bar{z} z}=0,
\end{aligned}
$$

where sum over $i$ and $j$ is assumed with $i, j \in\{1,2\}$. We define

$$
\begin{array}{ll}
X_{1}^{\mu}=X^{\mu}, & X_{2}^{\mu}=Y^{\mu}, \\
V_{\mu}^{1}=V_{\mu}, & V_{\mu}^{2}=U_{\mu}, \\
\Lambda=-\frac{1}{\alpha^{\prime}}\left(\begin{array}{ll}
1 & \lambda \\
\lambda & \beta
\end{array}\right) .
\end{array}
$$

The vectors $V^{\mu}$ and $U^{\mu}$ are fixed in the spacetime. For each pair of these vectors we have a CFT. The extra terms in (6) are total derivatives. Thus, we shall see that they do not affect the status of $T(z)$ and $\tilde{T}(\bar{z})$ as generators of conformal transformations. The field $\Phi=V_{\mu} X^{\mu}$ in (6) is linear dilaton. In the same way $\Phi^{\prime}=U_{\mu} Y^{\mu}$ is a linear field in the Y-space. In fact, by introducing the worldsheet fields $\left\{Y^{\mu}(\sigma, \tau)\right\}$ and defining the energy-momentum tensor (6), we have generalized the linear dilaton CFT. The case $\beta=1$ and $\lambda=0$ decomposes the model to two copies of the linear dilaton CFT.

\section{OPERATOR PRODUCT EXPANSIONS (OPES)}

\subsection{The OPEs $X X, X Y$ and $Y Y$}

We use the path integral formalism to derive operator equations. Since the path integral of a total derivative is zero, we have the equation

$$
\begin{aligned}
& 0=\int D X D Y \frac{\delta}{\delta X_{\mu}(z, \bar{z})}\left[e^{-S} X^{v}(w, \bar{w}) \ldots\right] \\
& =\int D X D Y e^{-S}\left[\left(\frac{1}{\pi \alpha^{\prime}} \partial_{z} \partial_{\bar{z}}\left[X^{\mu}(z, \bar{z})+\lambda Y^{\mu}(z, \bar{z})\right] X^{v}(w, \bar{w})+\eta^{\mu v} \delta^{(2)}(z-w, \bar{z}-\bar{w})\right) \ldots\right] \\
& =\left\langle\left(\frac{1}{\pi \alpha^{\prime}} \partial_{z} \partial_{\bar{z}}\left[X^{\mu}(z, \bar{z})+\lambda Y^{\mu}(z, \bar{z})\right] X^{v}(w, \bar{w})+\eta^{\mu v} \delta^{(2)}(z-w, \bar{z}-\bar{w})\right) \ldots\right\rangle .
\end{aligned}
$$

The point $(w, \bar{w})$ might be coincident with $(z, \bar{z})$, but the insertion "..." is arbitrary, which is away from $(z, \bar{z})$ and $(w, \bar{w})$. Arbitraryness of the insertion implies

$$
\begin{aligned}
& \frac{1}{\pi \alpha^{\prime}} \partial_{z} \partial_{\bar{z}}\left[X^{\mu}(z, \bar{z})+\lambda Y^{\mu}(z, \bar{z})\right] X^{v}(w, \bar{w}) \\
& =-\eta^{\mu v} \delta^{(2)}(z-w, \bar{z}-\bar{w}),
\end{aligned}
$$

as an operator equation. In the same way, the equation

$$
\int D X D Y \frac{\delta}{\delta X_{\mu}(z, \bar{z})}\left[e^{-S} Y^{v}(w, \bar{w}) \ldots\right]=0
$$

gives the operator equation

$$
\partial_{z} \partial_{\bar{z}}\left[X^{\mu}(z, \bar{z})+\lambda Y^{\mu}(z, \bar{z})\right] Y^{\nu}(w, \bar{w})=0 .
$$

In the equation (10) change $X_{\mu}$ to $Y_{\mu}$, we obtain

$$
\begin{aligned}
& \frac{1}{\pi \alpha^{\prime}} \partial_{z} \partial_{\bar{z}}\left[\lambda X^{\mu}(z, \bar{z})+\beta Y^{\mu}(z, \bar{z})\right] Y^{\nu}(w, \bar{w}) \\
& =-\eta^{\mu v} \delta^{(2)}(z-w, \bar{z}-\bar{w}) .
\end{aligned}
$$

Similarly, in the first line of (8) replacing $X_{\mu}$ by $Y_{\mu}$ leads to

$$
\partial_{z} \partial_{\bar{z}}\left[\lambda X^{\mu}(z, \bar{z})+\beta Y^{\mu}(z, \bar{z})\right] X^{\nu}(w, \bar{w})=0 .
$$


The equations (9), (11), (12) and (13) give the following equations

$$
\begin{aligned}
& \partial_{z} \partial_{\bar{z}} X^{\mu}(z, \bar{z}) X^{v}(w, \bar{w})=-\frac{\pi \beta \alpha^{\prime}}{\beta-\lambda^{2}} \eta^{\mu v} \delta^{(2)}(z-w, \bar{z}-\bar{w}), \\
& \partial_{z} \partial_{\bar{z}} Y^{\mu}(z, \bar{z}) X^{v}(w, \bar{w})=\frac{\pi \lambda \alpha^{\prime}}{\beta-\lambda^{2}} \eta^{\mu v} \delta^{(2)}(z-w, \bar{z}-\bar{w}), \\
& \partial_{z} \partial_{\bar{z}} X^{\mu}(z, \bar{z}) Y^{v}(w, \bar{w})=\frac{\pi \lambda \alpha^{\prime}}{\beta-\lambda^{2}} \eta^{\mu v} \delta^{(2)}(z-w, \bar{z}-\bar{w}) \\
& \partial_{z} \partial_{\bar{z}} Y^{\mu}(z, \bar{z}) Y^{v}(w, \bar{w})=-\frac{\pi \alpha^{\prime}}{\beta-\lambda^{2}} \eta^{\mu v} \delta^{(2)}(z-w, \bar{z}-\bar{w})
\end{aligned}
$$

That is, the equations of motion (4) hold except at the coincident point $(z, \bar{z})=(w, \bar{w})$. Define the matrix $Q_{i j}$ as in the following

$$
Q=\frac{\alpha^{\prime}}{2\left(\beta-\lambda^{2}\right)}\left(\begin{array}{cc}
\beta & -\lambda \\
-\lambda & 1
\end{array}\right)
$$

Thus, the equations (14) can be written in the compact form

$$
\partial_{z} \partial_{\bar{z}} X_{i}^{\mu}(z, \bar{z}) X_{j}^{v}(w, \bar{w})=-2 \pi Q_{i j} \eta^{\mu v} \delta^{(2)}(z-w, \bar{z}-\bar{w}) .
$$

According to this, we have the normal ordered equation

$$
: X_{i}^{\mu}(z, \bar{z}) X_{j}^{v}(w, \bar{w}):=X_{i}^{\mu}(z, \bar{z}) X_{j}^{v}(w, \bar{w})+Q_{i j} \eta^{\mu v} \ln |z-w|^{2},
$$

where $\partial_{z} \partial_{\bar{z}} \ln |z-w|^{2}=2 \pi \delta^{(2)}(z-w, \bar{z}-\bar{w})$ has been used. The equation (16) and (17) indicate the equation of motion

$$
\partial_{z} \partial_{\bar{z}}: X_{i}^{\mu}(z, \bar{z}) X_{j}^{v}(w, \bar{w}):=0 .
$$

\subsection{The $T T$ OPE}

Let $\mathcal{F}$ be any functional of $\left\{X^{\mu}\right\}$ and $\left\{Y^{\mu}\right\}$. Thus, the generalization of (17) is defined by

$$
\mathcal{F}[X, Y]=\exp \left(-\frac{1}{2} Q_{i j} \int d^{2} z_{1} d^{2} z_{2} \ln \left|z_{1}-z_{2}\right|^{2} \frac{\delta}{\delta X_{i}^{\mu}\left(z_{1}, \bar{z}_{1}\right)} \frac{\delta}{\delta X_{j \mu}\left(z_{2}, \bar{z}_{2}\right)}\right): \mathcal{F}[X, Y]:
$$

The OPE for any pair of the operators $\mathcal{F}$ and $\mathcal{G}$ is given by

$$
: \mathcal{F}:: \mathcal{G}:=\exp \left(-Q_{i j} \int d^{2} z_{1} d^{2} z_{2} \ln \left|z_{1}-z_{2}\right|^{2} \frac{\delta}{\delta X_{i F}^{\mu}\left(z_{1}, \bar{z}_{1}\right)} \frac{\delta}{\delta X_{\mu G}^{j}\left(z_{2}, \bar{z}_{2}\right)}\right): \mathcal{F} \mathcal{G}:
$$

where the functional derivatives act only on the fields $\mathcal{F}$ or $\mathcal{G}$, respectively. For $\mathcal{F}=X_{i}^{\mu}(z, \bar{z})$ and $\mathcal{G}=X_{j}^{v}(w, \bar{w})$ this reduces to the equation (17), as expected.

Using the equation (20), we obtain

$$
\begin{aligned}
& : \partial_{z} X_{i}^{\mu}(z) \partial_{z} X_{j \mu}(z):: \partial_{w} X_{k}^{v}(w) \partial_{w} X_{l v}(w):= \\
& : \partial_{z} X_{i}^{\mu}(z) \partial_{z} X_{j \mu}(z) \partial_{w} X_{k}^{v}(w) \partial_{w} X_{l v}(w):+\frac{D}{(z-w)^{4}}\left(Q_{i k} Q_{j l}+Q_{i l} Q_{j k}\right) \\
& -\frac{1}{(z-w)^{2}}\left(Q_{i k}: \partial_{z} X_{j}^{\mu}(z) \partial_{w} X_{l \mu}(w):+Q_{i l}: \partial_{z} X_{j}^{\mu}(z) \partial_{w} X_{k \mu}(w):\right. \\
& \left.+Q_{j k}: \partial_{z} X_{i}^{\mu}(z) \partial_{w} X_{l \mu}(w):+Q_{j l}: \partial_{z} X_{i}^{\mu}(z) \partial_{w} X_{k \mu}(w):\right)
\end{aligned}
$$

Now the Taylor expansion of $\partial_{z} X_{i}^{\mu}(z)$ around $z=w$ changes this equation to 


$$
\begin{aligned}
& : \partial_{z} X_{i}^{\mu}(z) \partial_{z} X_{j \mu}(z):: \partial_{w} X_{k}^{v}(w) \partial_{w} X_{l v}(w): \sim \frac{D}{(z-w)^{4}}\left(Q_{i k} Q_{j l}+Q_{i l} Q_{j k}\right) \\
& -\frac{1}{(z-w)^{2}}\left(Q_{i k}: \partial_{w} X_{j}^{\mu}(w) \partial_{w} X_{l \mu}(w):+Q_{i l}: \partial_{w} X_{j}^{\mu}(w) \partial_{w} X_{k \mu}(w):\right. \\
& \left.+Q_{j k}: \partial_{w} X_{i}^{\mu}(w) \partial_{w} X_{l \mu}(w):+Q_{j l}: \partial_{w} X_{i}^{\mu}(w) \partial_{w} X_{k \mu}(w):\right) \\
& -\frac{1}{z-w}\left(Q_{i k}: \partial_{w}^{2} X_{j}^{\mu}(w) \partial_{w} X_{l \mu}(w):+Q_{i l}: \partial_{w}^{2} X_{j}^{\mu}(w) \partial_{w} X_{k \mu}(w):\right. \\
& \left.+Q_{j k}: \partial_{w}^{2} X_{i}^{\mu}(w) \partial_{w} X_{l \mu}(w):+Q_{j l}: \partial_{w}^{2} X_{i}^{\mu}(w) \partial_{w} X_{k \mu}(w):\right)
\end{aligned}
$$

where the non-singular terms have been omitted. For calculating the $T T$ OPE we also need the following OPEs

$$
\begin{aligned}
& : \partial_{z} X_{i}^{v}(z) \partial_{z} X_{j v}(z): \partial_{w}^{2} X_{k}^{\mu}(w) \sim \\
& -\frac{2}{(z-w)^{3}}\left(Q_{i k} \partial_{w} X_{j}^{\mu}(w)+Q_{j k} \partial_{w} X_{i}^{\mu}(w)\right) \\
& -\frac{2}{(z-w)^{2}}\left(Q_{i k} \partial_{w}^{2} X_{j}^{\mu}(w)+Q_{j k} \partial_{w}^{2} X_{i}^{\mu}(w)\right) \\
& -\frac{1}{z-w}\left(Q_{i k} \partial_{w}^{3} X_{j}^{\mu}(w)+Q_{j k} \partial_{w}^{3} X_{i}^{\mu}(w)\right),
\end{aligned}
$$

$$
\begin{aligned}
& \partial_{z}^{2} X_{k}^{\mu}(z): \partial_{w} X_{i}^{v}(w) \partial_{w} X_{j v}(w): \\
& \sim \frac{2}{(z-w)^{3}}\left(Q_{k i} \partial_{w} X_{j}^{\mu}(w)+Q_{k j} \partial_{w} X_{i}^{\mu}(w)\right) \\
& \partial_{z}^{2} X_{i}^{\mu}(z) \partial_{w}^{2} X_{j}^{v}(w) \sim \frac{6}{(z-w)^{4}} \eta^{\mu v} Q_{i j} .
\end{aligned}
$$

Adding all these together we obtain the $T T$ OPE as in the following

$$
\begin{aligned}
& T(z) T(w) \sim \frac{c}{2(z-w)^{4}}-\frac{1}{(z-w)^{2}}\left[2 \Lambda_{i j} V_{\mu}^{k}\left(Q_{i k} \partial_{w}^{2} X_{j}^{\mu}(w)+Q_{j k} \partial_{w}^{2} X_{i}^{\mu}(w)\right)\right. \\
& +\Lambda_{i j} \Lambda_{k l}\left(Q_{i k} \partial_{w} X_{j \mu}(w) \partial_{w} X_{l}^{\mu}(w)+Q_{i l} \partial_{w} X_{j \mu}(w) \partial_{w} X_{k}^{\mu}(w)\right. \\
& \left.\left.+Q_{j k} \partial_{w} X_{i \mu}(w) \partial_{w} X_{l}^{\mu}(w)+Q_{j l} \partial_{w} X_{i \mu}(w) \partial_{w} X_{k}^{\mu}(w)\right)\right] \\
& -\frac{1}{z-w}\left[\Lambda _ { i j } \Lambda _ { k l } \left(Q_{i k} \partial_{w}^{2} X_{j \mu}(w) \partial_{w} X_{l}^{\mu}(w)+Q_{i l} \partial_{w}^{2} X_{j \mu}(w) \partial_{w} X_{k}^{\mu}(w)\right.\right. \\
& \left.+Q_{j k} \partial_{w}^{2} X_{i \mu}(w) \partial_{w} X_{l}^{\mu}(w)+Q_{j l} \partial_{w}^{2} X_{i \mu}(w) \partial_{w} X_{k}^{\mu}(w)\right) \\
& \left.+\Lambda_{i j} V_{\mu}^{k}\left(Q_{i k} \partial_{w}^{3} X_{j}^{\mu}(w)+Q_{j k} \partial_{w}^{3} X_{i}^{\mu}(w)\right)\right]
\end{aligned}
$$

The $\tilde{T} \tilde{T}$ OPE also has a similar form in terms of $\bar{z}, \bar{w}$ and $\tilde{c}$.

The central charges are given by

$$
\begin{aligned}
& c=\tilde{c}=2 D \Lambda_{i j} \Lambda_{k l}\left(Q_{i k} Q_{j l}+Q_{i l} Q_{j k}\right)+12 Q_{i j} V_{\mu}^{i} V_{j}^{\mu}, \\
& =2 D+\frac{6 \alpha^{\prime}}{\beta-\lambda^{2}}\left(\beta V_{\mu} V^{\mu}-2 \lambda V_{\mu} U^{\mu}+U_{\mu} U^{\mu}\right) .
\end{aligned}
$$

Vanishing conformal anomaly relates the parameters of the model. That is, string actually can move in a wide range of the dimensions. However, by adjusting the variables $\beta, \lambda, V^{\mu}$ and $U^{\mu}$, we can obtain desirable dimension for the spacetime. The case $V^{\mu}=U^{\mu}=0$ gives the central charges $c=\tilde{c}=2 D$, i.e., $D$ for $\left\{X^{\mu}\right\}$ and $D$ for $\left\{Y^{\mu}\right\}$. If $\beta=1$ and $\lambda=0$, the action
(1) reduces to two copies of the free string action, and hence the energy-momentum tensor (6) is modified to two copies of the energy-momentum tensor of the linear dilaton CFT. In this case the central charge also reduces to two copies of the central charge of the linear dilaton CFT

$$
\begin{aligned}
& c=c_{X}+c_{Y}, \\
& c_{X}=D+6 \alpha^{\prime} V_{\mu} V^{\mu}, \\
& c_{Y}=D+6 \alpha^{\prime} U_{\mu} U^{\mu} .
\end{aligned}
$$


Since there is $\Lambda Q=-\frac{1}{2} I_{2 \times 2}$, the $T T$ OPE (26), and similarly the $\tilde{T} \tilde{T}$ OPE take the standard forms

$$
\begin{aligned}
T(z) T(w) & \sim \frac{c}{2(z-w)^{4}}+\frac{2}{(z-w)^{2}} T(w)+\frac{1}{z-w} \partial_{w} T(w), \\
\tilde{T}(\bar{z}) \tilde{T}(\bar{w}) & \sim \frac{\tilde{c}}{2(\bar{z}-\bar{w})^{4}}+\frac{2}{(\bar{z}-\bar{w})^{2}} \tilde{T}(\bar{w})+\frac{1}{\bar{z}-\bar{w}} \partial_{\bar{w}} \tilde{T}(\bar{w}) .
\end{aligned}
$$

According to the central charge terms, $T$ and $\tilde{T}$ are not conformal tensors. Apart from these terms, (29) is the statement that $T(z)$ and $\tilde{T}(\bar{z})$ are conformal fields of the weights $(2,0)$ and $(0,2)$, respectively.

\subsection{The OPEs $T X, T Y, \tilde{T} X$ and $\tilde{T} Y$}

The OPE $T X_{k}^{\mu}$ is

$$
\begin{aligned}
& T(z) X_{k}^{\mu}(w, \bar{w}) \sim \frac{1}{(z-w)^{2}} V_{i}^{\mu} Q_{i k}-\frac{1}{z-w} \Lambda_{i j}\left[Q_{i k} \partial_{w} X_{j}^{\mu}(w)\right. \\
& \left.+Q_{j k} \partial_{w} X_{i}^{\mu}(w)\right] .
\end{aligned}
$$

Thus, for $k=1$ and $k=2$ we obtain

$$
\begin{aligned}
& T(z) X^{\mu}(w, \bar{w}) \sim \frac{1}{(z-w)^{2}} \frac{\alpha^{\prime}}{2\left(\beta-\lambda^{2}\right)}\left(\beta V^{\mu}-\lambda U^{\mu}\right)+\frac{1}{z-w} \partial_{w} X^{\mu}(w), \\
& T(z) Y^{\mu}(w, \bar{w}) \sim \frac{1}{(z-w)^{2}} \frac{\alpha^{\prime}}{2\left(\beta-\lambda^{2}\right)}\left(-\lambda V^{\mu}+U^{\mu}\right)+\frac{1}{z-w} \partial_{w} Y^{\mu}(w) .
\end{aligned}
$$

In the same way we have

$$
\begin{aligned}
& \tilde{T}(\bar{z}) X^{\mu}(w, \bar{w}) \sim \frac{1}{(\bar{z}-\bar{w})^{2}} \frac{\alpha^{\prime}}{2\left(\beta-\lambda^{2}\right)}\left(\beta V^{\mu}-\lambda U^{\mu}\right)+\frac{1}{\bar{z}-\bar{w}} \partial_{\bar{w}} X^{\mu}(\bar{w}), \\
& \tilde{T}(\bar{z}) Y^{\mu}(w, \bar{w}) \sim \frac{1}{(\bar{z}-\bar{w})^{2}} \frac{\alpha^{\prime}}{2\left(\beta-\lambda^{2}\right)}\left(-\lambda V^{\mu}+U^{\mu}\right)+\frac{1}{\bar{z}-\bar{w}} \partial_{\bar{w}} Y^{\mu}(\bar{w}) .
\end{aligned}
$$

The $U$-terms and $V$-terms imply that $X^{\mu}$ and $Y^{\mu}$ are not conformal tensor operators. Putting away these terms (the square singular terms) of the above OPEs leads to the conditions

$$
\begin{aligned}
& \beta V^{\mu}-\lambda U^{\mu}=0, \\
& -\lambda V^{\mu}+U^{\mu}=0 .
\end{aligned}
$$

Since we assumed $\beta-\lambda^{2} \neq 0$, we obtain $V^{\mu}=U^{\mu}=0$. Therefore, (31) and (32) reduce to the OPEs $T^{\prime} X, T^{\prime} Y, \tilde{T}^{\prime} X$ and $\tilde{T}^{\prime} Y$. That is, with $T^{\prime}$ and $\tilde{T}^{\prime}$ the fields $X^{\mu}$ and $Y^{\mu}$ are conformal tensors, as expected. However, we shall not consider the case (33).

\section{CONFORMAL TRANSFORMATIONS OF $X^{\mu}$ AND $Y^{\mu}$}

The infinitesimal conformal transformations $z \rightarrow z^{\prime}=z+$ $\varepsilon g(z)$ and $\bar{z} \rightarrow \bar{z}^{\prime}=\bar{z}+\varepsilon g(z)^{*}$ imply the currents

$$
\begin{aligned}
& j(z)=i g(z) T(z), \\
& \tilde{j}(\bar{z})=i g(z)^{*} \tilde{T}(\bar{z}) .
\end{aligned}
$$

For any holomorphic function $g(z)$ these are conserved. These currents lead to the Ward identity

$$
\delta X_{i}^{\mu}(w, \bar{w})=-\varepsilon\left(\operatorname{Res}_{z \rightarrow w} g(z) T(z) X_{i}^{\mu}(w, \bar{w})+\operatorname{Re}_{\bar{z} \rightarrow \bar{w}} g(z)^{*} \tilde{T}(\bar{z}) X_{i}^{\mu}(w, \bar{w})\right)
$$

where "Res" and "Res" are coefficients of $(z-w)^{-1}$ and $(\bar{z}-$ $\bar{w})^{-1}$, respectively. From the OPEs (31) and (32) and the
Ward identity (35) we obtain the conformal transformations

$$
\begin{gathered}
\delta X^{\mu}(w, \bar{w})=-\varepsilon\left[g(w) \partial_{w} X^{\mu}(w)+g(w)^{*} \partial_{\bar{w}} X^{\mu}(\bar{w})\right] \\
-\frac{\alpha^{\prime} \varepsilon}{2\left(\beta-\lambda^{2}\right)}\left(\beta V^{\mu}-\lambda U^{\mu}\right)\left[\partial_{w} g(w)+\partial_{\bar{w}} g(w)^{*}\right]
\end{gathered}
$$




$$
\begin{array}{r}
\delta Y^{\mu}(w, \bar{w})=-\varepsilon\left[g(w) \partial_{w} Y^{\mu}(w)+g(w)^{*} \partial_{\bar{w}} Y^{\mu}(\bar{w})\right] \\
-\frac{\alpha^{\prime} \varepsilon}{2\left(\beta-\lambda^{2}\right)}\left(-\lambda V^{\mu}+U^{\mu}\right)\left[\partial_{w} g(w)+\partial_{\bar{w}} g(w)^{*}\right] .
\end{array}
$$

Due to the inhomogeneous parts, originated from $V^{\mu}, U^{\mu}, \beta$ and $\lambda$, the fields $X^{\mu}$ and $Y^{\mu}$ do not transform as conformal tensor. These parts also indicate that these transformations are not infinitesimal coordinate transformations $\delta z=\varepsilon g(z)$ and $\delta \bar{z}=\varepsilon g(z)^{*}$.

\section{MODE EXPANSIONS}

Now we express some quantities of the model in terms of the oscillators of $X^{\mu}$ and $Y^{\mu}$. The OPEs (31) and (32) give

$$
\begin{aligned}
& T(z) \partial_{w} X^{\mu}(w) \sim \frac{1}{(z-w)^{3}} \frac{\alpha^{\prime}}{\beta-\lambda^{2}}\left(\beta V^{\mu}-\lambda U^{\mu}\right)+\frac{1}{(z-w)^{2}} \partial_{w} X^{\mu}(w)+\frac{1}{z-w} \partial_{w}^{2} X^{\mu}(w), \\
& \tilde{T}(\bar{z}) \partial_{w} X^{\mu}(w) \sim 0,
\end{aligned}
$$

$$
\begin{aligned}
& T(z) \partial_{w} Y^{\mu}(w) \sim \frac{1}{(z-w)^{3}} \frac{\alpha^{\prime}}{\beta-\lambda^{2}}\left(-\lambda V^{\mu}+U^{\mu}\right) \\
& +\frac{1}{(z-w)^{2}} \partial_{w} Y^{\mu}(w)+\frac{1}{z-w} \partial_{w}^{2} Y^{\mu}(w) \\
& \tilde{T}(\bar{z}) \partial_{w} Y^{\mu}(w) \sim 0 .
\end{aligned}
$$

Thus, the conformal weights of $\partial_{z} X^{\mu}(z)$ and $\partial_{z} Y^{\mu}(z)$ are

$$
\begin{array}{ll}
h_{\partial X}=1 & , \quad \tilde{h}_{\partial X}=0, \\
h_{\partial Y}=1 & \tilde{h}_{\partial Y}=0 .
\end{array}
$$

According to these conformal weights, we obtain the Laurent expansions

$$
\begin{aligned}
& \partial_{z} X_{i}^{\mu}(z)=-i \sqrt{\frac{\alpha^{\prime}}{2}} \sum_{m=-\infty}^{\infty} \frac{\alpha_{(i) m}^{\mu}}{z^{m+1}}, \\
& \partial_{\bar{z}} X_{i}^{\mu}(\bar{z})=-i \sqrt{\frac{\alpha^{\prime}}{2}} \sum_{m=-\infty}^{\infty} \frac{\tilde{\alpha}_{(i) m}^{\mu}}{\bar{z}^{m+1}} .
\end{aligned}
$$

Single-valuedness of $X^{\mu}$ and $Y^{\mu}$ implies that

$$
\alpha_{(i) 0}^{\mu}=\tilde{\alpha}_{(i) 0}^{\mu}=\sqrt{\frac{\alpha^{\prime}}{2}} p_{i}^{\mu},
$$

where $p_{i}^{\mu}$ is the linear momentum. Now integration of the expansions (41) gives the closed string solution

$$
\begin{aligned}
& X_{i}^{\mu}(z, \bar{z})=x_{i}^{\mu}-i \frac{\alpha^{\prime}}{2} p_{i}^{\mu} \ln |z|^{2}+i \sqrt{\frac{\alpha^{\prime}}{2}} \sum_{m \neq 0}^{\infty} \frac{1}{m} \\
& \times\left(\frac{\alpha_{(i) m}^{\mu}}{z^{m}}+\frac{\tilde{\alpha}_{(i) m}^{\mu}}{\bar{z}^{m}}\right) .
\end{aligned}
$$

Reality of $X_{i}^{\mu}$ implies that $\alpha_{(i) m}^{\mu \dagger}=\alpha_{(i)(-m)}^{\mu}$ and $\tilde{\alpha}_{(i) m}^{\mu \dagger}=$ $\tilde{\alpha}_{(i)(-m)}^{\mu}$.

The expansions (41) also lead to

$$
\begin{aligned}
& \alpha_{(i) m}^{\mu}=\sqrt{\frac{2}{\alpha^{\prime}}} \oint_{C} \frac{d z}{2 \pi} z^{m} \partial_{z} X_{i}^{\mu}(z), \\
& \tilde{\alpha}_{(i) m}^{\mu}=-\sqrt{\frac{2}{\alpha^{\prime}}} \oint_{\tilde{C}} \frac{d \bar{z}}{2 \pi} \bar{z}^{m} \partial_{\bar{z}} X_{i}^{\mu}(\bar{z}),
\end{aligned}
$$

where $C$ in the $z$-plane and $\tilde{C}$ in the $\bar{z}$-plane are counterclockwise. Therefore, by using the OPEs $\partial_{z} X_{i}^{\mu}(z) \partial_{w} X_{j}^{v}(w)$ and $\partial_{\bar{z}} X_{i}^{\mu}(\bar{z}) \partial_{\bar{w}} X_{j}^{v}(\bar{w})$ we obtain

$$
\begin{aligned}
& {\left[\alpha_{(i) m}^{\mu}, \alpha_{(j) n}^{v}\right]=\left[\tilde{\alpha}_{(i) m}^{\mu}, \tilde{\alpha}_{(j) n}^{v}\right]=\frac{2}{\alpha^{\prime}} m \eta^{\mu v} Q_{i j} \delta_{m,-n},} \\
& {\left[\alpha_{(i) m}^{\mu}, \tilde{\alpha}_{(j) n}^{v}\right]=0,} \\
& {\left[x_{i}^{\mu}, p_{j}^{v}\right]=\frac{2 i}{\alpha^{\prime}} \eta^{\mu \nu} Q_{i j} .}
\end{aligned}
$$

For $\lambda \neq 0$ we observe that the oscillators of $X^{\mu}$ do not commute with the oscillators of $Y^{\mu}$.

In terms of the oscillators the nonzero elements of the energy-momentum tensor find the forms

$$
\begin{aligned}
& T(z)=-\frac{\alpha^{\prime}}{2} \eta_{\mu v} \Lambda_{i j} \sum_{m=-\infty}^{\infty} \sum_{n=-\infty}^{\infty} \frac{1}{z^{m+n+2}}: \alpha_{(i) m}^{\mu} \alpha_{(j) n}^{v}:+i \sqrt{\frac{\alpha^{\prime}}{2}} V_{\mu}^{i} \sum_{m=-\infty}^{\infty} \frac{m+1}{z^{m+2}} \alpha_{(i) m}^{\mu}, \\
& \tilde{T}(\bar{z})=-\frac{\alpha^{\prime}}{2} \eta_{\mu v} \Lambda_{i j} \sum_{m=-\infty}^{\infty} \sum_{n=-\infty}^{\infty} \frac{1}{\bar{z}^{m+n+2}}: \tilde{\alpha}_{(i) m}^{\mu} \tilde{\alpha}_{(j) n}^{\nu}:+i \sqrt{\frac{\alpha^{\prime}}{2}} V_{\mu}^{i} \sum_{m=-\infty}^{\infty} \frac{m+1}{\bar{z}^{m+2}} \tilde{\alpha}_{(i) m}^{\mu} .
\end{aligned}
$$


The Virasoro operators are

$$
\begin{aligned}
L_{m} & =\oint_{C} \frac{d z}{2 \pi i} z^{m+1} T(z), \\
\tilde{L}_{m} & =\oint_{\tilde{C}} \frac{d \bar{z}}{2 \pi i} \bar{z}^{m+1} \tilde{T}(\bar{z}) .
\end{aligned}
$$

In terms of the oscillators they take the forms

$$
\begin{aligned}
& L_{m}=-\frac{\alpha^{\prime}}{2} \Lambda_{i j} \eta_{\mu v} \sum_{n=-\infty}^{\infty}: \alpha_{(i) m-n}^{\mu} \alpha_{(j) n}^{v}:+i \sqrt{\frac{\alpha^{\prime}}{2}}(m+1) V_{\mu}^{i} \alpha_{(i) m}^{\mu}, \\
& \tilde{L}_{m}=-\frac{\alpha^{\prime}}{2} \Lambda_{i j} \eta_{\mu v} \sum_{n=-\infty}^{\infty}: \tilde{\alpha}_{(i) m-n}^{\mu} \tilde{\alpha}_{(j) n}^{v}:+i \sqrt{\frac{\alpha^{\prime}}{2}}(m+1) V_{\mu}^{i} \tilde{\alpha}_{(i) m}^{\mu}
\end{aligned}
$$

Using the standard methods one can show that the normal ordering constant for all $L_{m}$ and $\tilde{L}_{m}$ is zero. According to the equations (45), or the standard form of the OPEs $T T$ and $\tilde{T} \tilde{T}$, i.e. the equations (29), the Virasoro algebra also has the standard form

$$
\begin{aligned}
& {\left[L_{m}, L_{n}\right]=(m-n) L_{m+n}+\frac{c}{12}\left(m^{3}-m\right) \delta_{m,-n},} \\
& {\left[\tilde{L}_{m}, \tilde{L}_{n}\right]=(m-n) \tilde{L}_{m+n}+\frac{\tilde{c}}{12}\left(m^{3}-m\right) \delta_{m,-n},} \\
& {\left[L_{m}, \tilde{L}_{n}\right]=0 .}
\end{aligned}
$$

The Hamiltonian of the system is given by

$$
H=L_{0}+\tilde{L}_{0}-\frac{c+\tilde{c}}{24}
$$

Thus, the equations (27) and (48) express this Hamiltonian in terms of the oscillators and the parameters of the model

$$
\begin{aligned}
H=\quad & \frac{1}{2} \alpha^{\prime}\left(p_{1} \cdot p_{1}+2 \lambda p_{1} \cdot p_{2}+\beta p_{2} \cdot p_{2}+2 i V \cdot p_{1}+2 i U \cdot p_{2}\right) \\
& -\alpha^{\prime} \Lambda_{i j} \eta_{\mu v} \sum_{n=1}^{\infty}\left(\alpha_{(i)(-n)}^{\mu} \alpha_{(j) n}^{v}+\tilde{\alpha}_{(i)(-n)}^{\mu} \tilde{\alpha}_{(j) n}^{v}\right) \\
& -\frac{\alpha^{\prime}}{2\left(\beta-\lambda^{2}\right)}(\beta V . V-2 \lambda V . U+U . U)-\frac{D}{6}
\end{aligned}
$$

where the symmetry of $\eta_{\mu v}$ and $\Lambda_{i j}$ were introduced.

For the open string there are

$$
\alpha_{(i) m}^{\mu}=\tilde{\alpha}_{(i) m}^{\mu}, \quad \alpha_{(i) 0}^{\mu}=\tilde{\alpha}_{(i) 0}^{\mu}=\sqrt{2 \alpha^{\prime}} p_{i}^{\mu},
$$

and hence the solution is

$X_{i}^{\mu}(z, \bar{z})=x_{i}^{\mu}-i \alpha^{\prime} p_{i}^{\mu} \ln |z|^{2}+i \sqrt{\frac{\alpha^{\prime}}{2}} \sum_{m \neq 0}^{\infty} \frac{\alpha_{(i) m}^{\mu}}{m}\left(\frac{1}{z^{m}}+\frac{1}{\bar{z}^{m}}\right)(53)$

The corresponding energy-momentum tensor and Virasoro operators are given by the first equations of (46) and (48). Thus, the associated Virasoro algebra also is described by the first equation of (49).

Note that we imposed the boundary conditions of the closed string and open string on $Y^{\mu}(\sigma, \tau)$. However,
$Y^{\mu}(\sigma, \tau)$ may be neither closed nor open. Assuming closeness or openness for $Y^{\mu}(\sigma, \tau)$, the worldsheet fields $\left(X^{\mu}(\sigma, \tau), Y^{\mu}(\sigma, \tau)\right)$ find four configurations: (closed , closed), (open, open), (open, closed) and (closed, open). We considered the first and the second cases. The third and the fourth cases also can be investigated in the same way.

\section{CONCLUSIONS AND SUMMARY}

We studied a CFT model with two kinds of the bosonic degrees of freedom $X^{\mu}$ and $Y^{\mu}$, which interact kinetically with each other. For each kind of these fields we introduced a linear dilaton field.

Using the path integral formalism, we obtained the OPEs $X X, X Y$ and $Y Y$. These OPEs enabled us to introduce a general definition for the OPEs. We observed that the $T T$ and $\tilde{T} \tilde{T}$ OPEs of the model have the standard forms. Due to the vectors $V^{\mu}$ and $U^{\mu}$, which define the dilatons, the OPEs $T X$, $T Y, \tilde{T} X$ and $\tilde{T} Y$ have deviations from the standard forms of them. The central charge of the model depends on the spacetime dimension, the parameters of the theory and the vectors $V^{\mu}$ and $U^{\mu}$. A vanishing conformal anomaly and hence a desirable dimension for the spacetime can be achieved by tuning these variables.

Putting away the interacting terms of the action, the model split into two copies of the linear dilaton CFT. The splitting also occurs for the energy-momentum tensor and hence for the central charge.

Using the conserved currents, associated to the conformal symmetry, the conformal transformations of the fields $X^{\mu}$ and $Y^{\mu}$ have been extracted. Therefore, the vectors $V^{\mu}$ and $U^{\mu}$ and also the parameters of the model indicate that $X^{\mu}$ and $Y^{\mu}$ are not conformal tensors. That is, these transformations are not pure coordinate transformations.

According to the mode expansions of $X^{\mu}$ and $Y^{\mu}$, we obtained the oscillator-algebra of the model. Due to the nonzero coupling constant $\lambda$ the oscillators of $X^{\mu}$ do not commute with the oscillators of $Y^{\mu}$. We expressed the energymomentum tensor and the Virasoro operators in terms of the oscillators. We observed that the Virasoro operators also form the standard algebra. 
[1] T. Takayanagi, JHEP 0412 (2004) 071, hep-th/0411019; JHEP 0509 (2005) 001, hep-th/0507065.

[2] S. Nakamura and V. Niarchos, JHEP 10 (2005) 025.

[3] O. Bergman and S. Hirano, Nucl. Phys. 744 (2006) 136.

[4] Y. Hikida, K. Hosomichi and Y. Sugawara, Nucl. Phys. B589
(2000) 134.

[5] P.M. Ho, S.Y. Darren Shih, JHEP 0801 (2008) 054, arXiv: 0711.2792.

[6] M. Li, Phys. Rev. D54 (1996) 1644, hep-th/9512042. 